\title{
A simple standard technique for labyrinthectomy in the rat: A methodical communication with a detailed description of the surgical process
}

\author{
GL Nádasy ${ }^{1,2}$, G Raffai $^{1}$, E Fehér $^{3}$, G Schaming $^{1}$, E Monos ${ }^{1}$ \\ ${ }^{1}$ Experimental Research Department and Department of Human Physiology, Semmelweis University, \\ Budapest, Hungary \\ ${ }^{2}$ Department of Physiology, Semmelweis University, Budapest, Hungary \\ ${ }^{3}$ Department of Anatomy, Histology and Embryology, Semmelweis University, Budapest, Hungary
}

Received: July 3, 2015

Accepted: July 4, 2016

\begin{abstract}
Aims: Labyrinthectomized rats are suitable models to test consequences of vestibular lesion and are widely used to study neural plasticity. We describe a combined microsurgical-chemical technique that can be routinely performed with minimum damage. Methods: Caudal leaflet of the parotis is elevated. The tendinous fascia covering the bulla is opened frontally from the sternomastoid muscle's tendon while sparing facial nerve branches. A 4 mm diameter hole is drilled into the bulla's hind lower lateral wall to open the common (in rodents) mastoid-tympanic cavity. The cochlear crista (promontory) at the lower posterior part of its medial wall is identified as a bony prominence. A $1 \mathrm{~mm}$ diameter hole is drilled into its lower part. The perilymphatic/endolymphatic fluids with tissue debris of the Corti organ are suctioned. Ethanol is injected into the hole. Finally, $10 \mu \mathrm{L}$ of sodium arsenite solution $(50 \mu \mathrm{M} / \mathrm{mL})$ is pumped into the labyrinth and left in place for $15 \mathrm{~min}$. Simple closure in two layers (fascia and skin) is sufficient. Results and conclusion: All rats had neurological symptoms specific for labyrinthectomy (muscle tone, body position, rotatory movements, nystagmus, central deafness). Otherwise, their behavior was unaffected, drinking and eating normally. After a few days, they learned to balance relying on visual and somatic stimuli (neuroplasticity).
\end{abstract}

Keywords: rat, labyrinthectomy, vestibular organ, neural plasticity, experimental surgery

\section{Introduction}

Labyrinthectomy in laboratory rodents is a crucial technique in sensory physiology, in studies on gravitational stress, microgravity effects, and in other fields of neurobiology. Its significance has obtained a new impetus as neuronal compensation after labyrinthectomy was recognized as an effective model to study the diverse (physiological, cellular, and molecular) processes leading to neuronal plasticity $(2,5,10,14,15,17,18,20,21)$. Neural plasticity is one key process in potential recovery after stroke (8). Most surgical techniques reported until now include extensive extirpation of the wall of the meatus acusticus externus and structures of middle ear $(1,4,7,9,12,15,16,22,25)$. The specific anatomy of the rodent skull, however, provides an alternative surgical approach through the tympanic bulla $(6,10,11,13)$. No publication, however, is available, with the exception of Hitier et al. (11) with a

Corresponding author: GL Nádasy, MD, PhD, Med Habil, Sen. Assoc. Prof. Physiology

Department of Physiology, Semmelweis University

Room 3.317, Túzoltó u. 37-47, H-1094 Budapest, Hungary

Phone: +36 12100290 ext. 60317; Cellular phone: +36120586 1406; Fax: +361334 3162; Email: nadasy. gyorgy@med.semmelweis-univ.hu 
sufficiently detailed description on how to expose the rat vestibular cavity surgically, while inducing minimum damage to the surrounding sensitive neck, temporal, and skull structures. In frame of our studies on the effect of gravitation load on the venous system (19), we have developed a combined microsurgical-chemical labyrinthectomy technique in the rat with even less structural damage than provided by any of the earlier techniques. It ensures the preservation of all other important structures and provides the animals with normal behavior with the exception of the effects of labyrinthine lesion. It can be relatively, easily, reproducibly executed, and standardized, and thus, it could ensure an even wider application for this already popular technique.

The purpose of this paper was to describe this technique in sufficient for reproduction detail.

\section{Materials and Methods}

The animal procedures included in this paper conform with the Revised Guide for the Care and Use of Laboratory Animals (NIH-ILAR 1996), with the Hungarian Law on Animal Care (1998, Permission No. 36/1999 and 22.1/2960/003/2009) and were accepted by the Animal Care Committee of the Semmelweis University.

After continuous improvement of our technique (all of them yielding sufficient results but, according to our opinion, with more than necessary tissue damage), we tested our improved technique on 30 animals. Unilateral labyrinthectomy was made on 11 animals, while bilateral on the remaining animals. The weight of the male Sprague-Dawley rats was between 250 and $350 \mathrm{~g}$.

In Nembutal anesthesia (45 mg/kg i.p.), a skin incision, 25-30 mm long, is made on the neck. It runs from the dorsocaudal to the rostro-ventro-medial direction, a few millimeters behind and below the root of the earlobe (Fig. 1a). The preparation initially goes caudally below the skin. The caudal lobe of the parotis positioned in-between the layers of the plathysma will be prepared around and elevated from its base. Then, the preparation is continued in the rostral direction further elevating the caudal lobe of the parotis from its muscular base. Vascularization and the tubular system of the parotis thus remain intact. The underlying muscle surface will be cleared in the rostral direction including a small part of the masseter muscle. A branch of the facial nerve exiting from the muscle layers has to be cared for. Preparation of deeper muscles can begin at the hind edge of the masseter muscle, just behind and below the meatus acusticus externus and earlobe. The location of the mastoid bulla can be palpated: when positioning our index finger on the hind edge of the ramus of the mandible, pushing it toward the meatus acusticus externus, the tip palpates the bulla. As a useful orientation point, we have to look for is the characteristic small, white, shiny tendon of the sternomastoid muscle (Figs $1 \mathrm{~b}$ and $2 \mathrm{a}$ ). It originates from a small bony prominence on the cranial base, just behind the bulla. Its tiny muscular stomach covers the hind portion of the bulla. The fascia covering the mastoid bulla will be exposed rostrally. Somewhat more ventrally, where the exiting fibers of the facial nerve are less endangered, we can prepare underneath the sternomastoid muscle. The smooth, bony surface of the bulla can now be palpated with a blunt forceps through the tough, fibrous fascia covering it. Using the preparation microscope, groups of fibers of the facial nerve running rostrally and ventrally attached to this fascia can be identified. Then, a 1.5-2 mm cut is done in the fascia with microscissors at the lower hind part of the lateral surface of the bulla, between the two groups of facial nerve fibers. The direction of the cut is from the dorsocaudal toward the 

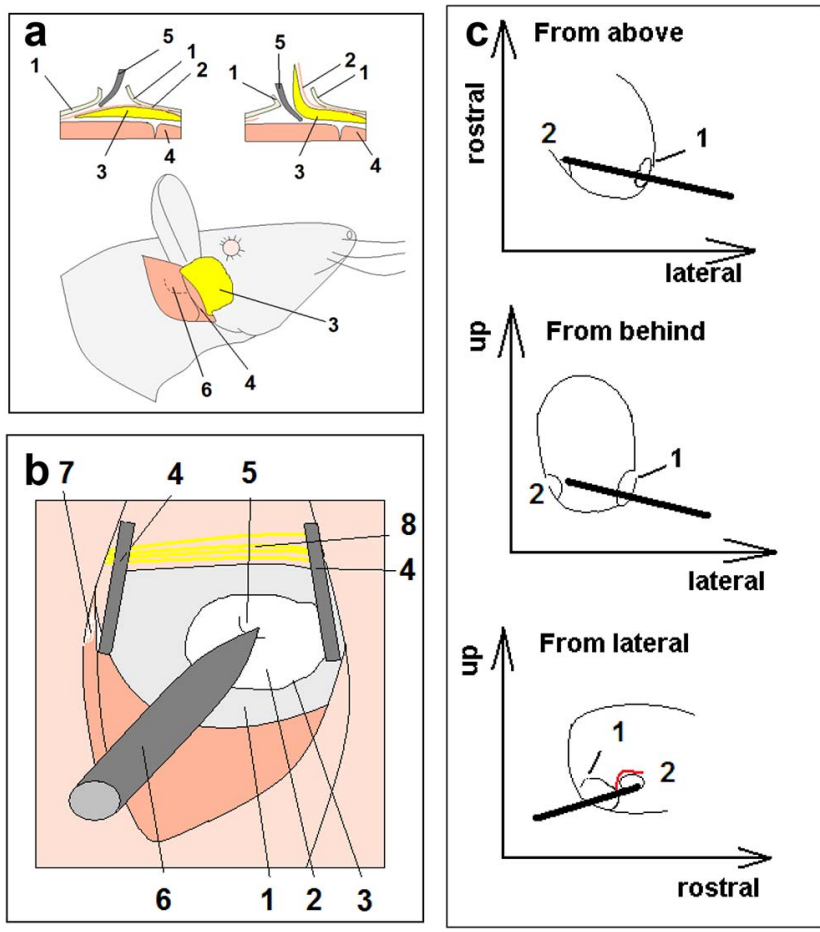

Fig. 1. Surgical process of rat labyrinthectomy. (a) Preparation of superficial layers: $1=$ skin, $2=$ superficial fascia, $3=$ hind lobe of the parotid gland, $4=$ masseter muscle, $5=$ forceps, and $6=$ location of the mastoid bulla beneath the muscle layer. (b) Drilling into the cochlear crista (promontory): $1=$ the smooth, shiny-bony surface of the cleared mastoid bulla, $2=$ hole, $3-4 \mathrm{~mm}$ diameter drilled into the hind lower lateral surface of the mastoid bulla, $3=$ edge of the hole drilled into the mastoid bulla, $4=$ microsurgery tissue retractor, $5=$ cochlear crista (promontory), $6=$ driller and $7=$ the white, shiny tendon of the sternomastoid muscle, an important orientation point, and $8=$ branches of the facial nerve, they should be spared. (c) 3-D positioning of the driller to bore the cochlear crista (promontory): $1=$ opening in the wall of the mastoid bulla and $2=$ the bony prominence of the cochlear crista (promontory). In the bottom panel, the course of the tympanic artery is shown above and behind the cochlear promontory, a massive artery in the rat, it should be spared

ventro-rostral direction. A small, curved, blunt pair of forceps is introduced into the cut and it is bluntly widened, minimizing this way the damage to the facial nerve fibers running around. The smooth, shiny, occipito-ventro-lateral surface of the mastoid bulla will be exposed (Figs $1 \mathrm{~b}$ and $2 \mathrm{a}$ ). While the retractors are positioned, care should be taken not to hurt the branches of the facial nerve (dorsally and caudally), the retroauricular artery branch (rostrally), and the larger branches of the external carotid artery (below the bulla and beneath the dorsal stomach of the biventer cervicis muscle). A larger microsurgical retractor (e.g., 17008-07, Fine Scientific Tools, Foster City, CA, USA) is used for the skin, plathysma, parotis, and more superficial muscles. Some might favor its application in an earlier phase of preparation. One has to be careful with the retractor application, as overextension of the tissues around the larynx might interfere with breathing of the animals. A smaller microsurgical retractor (17005-04, Fine Scientific Tools) should be applied to expose the immediate bony surface of the bulla (Figs $1 \mathrm{~b}$ and $2 \mathrm{a}$ ). Using a battery-supplied small driller, equipped with a spherical head of approximately $2 \mathrm{~mm}$ diameter, covered with diamond dust, a hole, with a diameter of approximately $4 \mathrm{~mm}$ was drilled into the posterior-lateral-ventral surface of the 

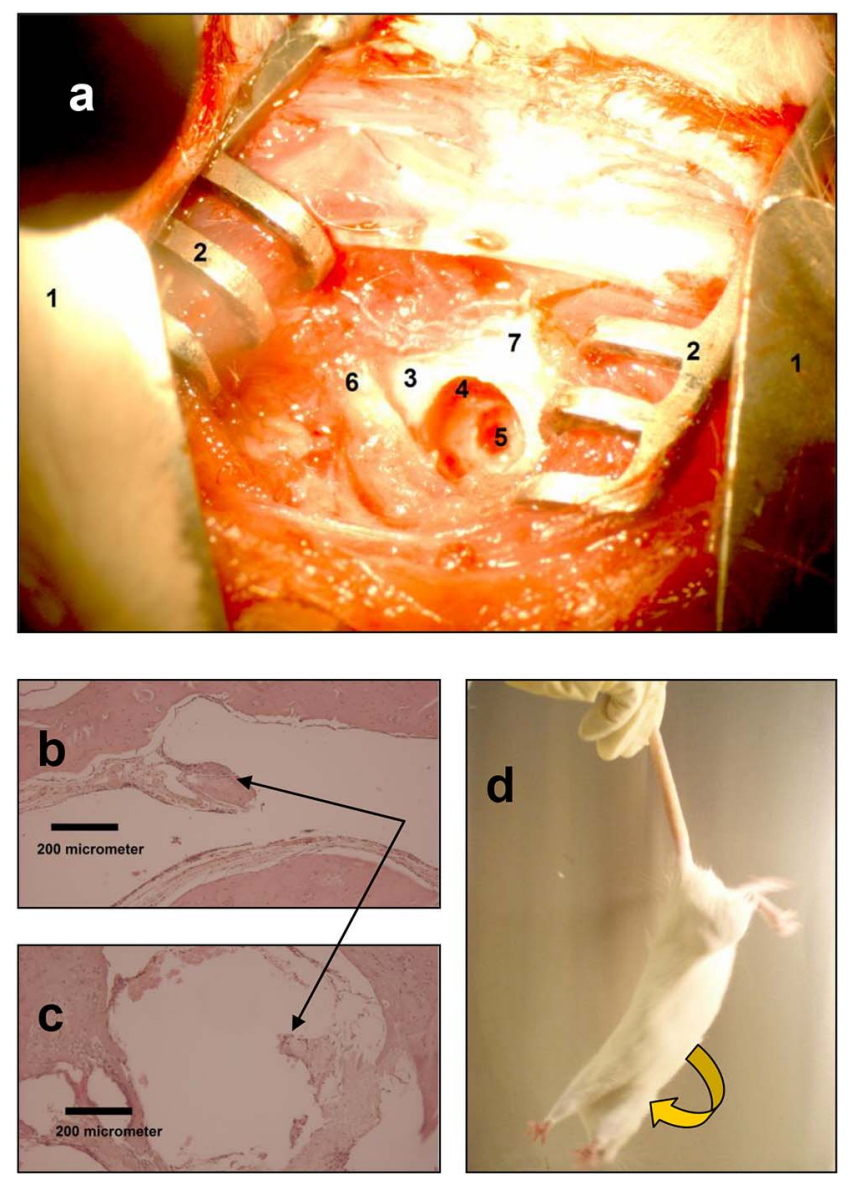

Fig. 2. Labyrinthectomy and its results. (a) Surgical situation with drilled cochlea: $1=$ large tissue retractor, 2 = fine tissue retractor, $3=$ mastoid bulla, $4=$ edge of hole drilled into the bulla, $5=$ hole drilled into the cochlear crista, $6=$ the white, shiny tendon of sternomastoid muscle, a convenient site for orientation. Right side ear, side view, rostral direction to the right, and $7=$ exit of facial nerve branches, they should be spared. (b) and (c). Decalcified histological section through the ampulla of intact (b) and ectomyzed

(c) labyrinth. Note: missing cupula and loss of cellularity in receptor cell layer at operated side (arrows). (d). Typical vestibular rotation of animals in direction of vestibular lesion

mastoid bulla. Thus, the common (in the rat) cavity of the middle ear with the mastoid bulla will be opened. With careful positioning of the head of the animal, of the microscope tube axis and of the fiberoptic illuminator, we bring into the visual field a bony prominence caused by the presence of the cochlea in the pyramid bone on the hind-medial surface of the mastoidtympanic cavity (Figs 1c and 2a). Depending on the exact location of the hole on the mastoid bulla, microscope optical axis angles of 20-40 degrees with the frontal plane of the head of the animal, occipitally from it, and 10-15 degrees with the horizontal plane of the head (below and over it; should be repeatedly tested) proved to be optimal. The crista (cochlear promontory) is located on the opposite, medial wall of the common cavity of the middle ear and of the mastoid bulla (Figs 1c and 2a). If we do not succeed in finding this, the hole on the lateral wall can be further enlarged in the required direction, but care has to be taken not to hurt the important structures. When the cochlear prominence has been positively identified (it forms a section of a sphere), it has to be drilled at its lower rostral part. This helps avoid bleeding from the tympanic artery, a massive vessel in the rat running superiorly and then posteriorly from the crista (cochlear promontory). The drilling can be done with a sharpedged head, with a diameter of about $1 \mathrm{~mm}$, also covered with diamond dust. The depth of drilling should not exceed $1 \mathrm{~mm}$ and the pressure on the driller has to be carefully controlled 
to avoid basal skull fracture and cerebrospinal fluid leakage to occur. We have to be careful to avoid any centrifugal motion of the driller's head driven by its rotation. Clear watery solution (endolymph and perilymph), sometimes mixed with lines of blood, will ooze from the hole. By carefully widening it, the typical scalar structure of the cochlea can be recognized. Then, the cochlear cavity is washed with saline and its contents aspired repeatedly as suggested by several authors $(1,3,4,10,13,14,24,25)$. Typical cellular debris of the Corti organ will appear in the suction fluid. Following this, multiple washes with concentrated ethanol will be done by pumping the ethanol into the labyrinth and suctioning it back (1). Finally, in a modified version of the technique advised by Matsuda et al. (18) and Vignaux et al. (23), $10 \mu \mathrm{L}$ of arsenite solution (sodium arsenite aqueous solution, Merck, Darmstadt, Germany, Budapest, Hungary, $50 \mu \mathrm{M} / \mathrm{mL}$ ) will be pumped into the opened labyrinthine cavity with a micro-injector syringe. Fifteen minutes is left for the toxic material to diffuse into the more hidden parts of the labyrinth. Fascia and skin will be closed in two layers. As a part of the postoperational care, a single shot of 300,000 IU penicillin is given intramuscularly against infections. Painkillers and tranquillizers should be chosen not to interfere with the ongoing neurology experiment. Success of labyrinthectomy can be judged from neurological symptoms $(5,18)$ and from decalcified histological sections through the pyramidal bone.

The animals were allowed to eat and drink freely even the night before the operation. No animal was lost as a result of aspiration (no laryngeal intubation was applied). Infusions should not be given, which simplifies the operational and postoperational care (no venous catheters were needed). The animals were never shown the signs of exsiccosis. Because of the carefully planned and executed surgery, neck tissue damage was kept at a minimum and recovery of the animals was fast. After some initial loss of body weight, they started to put on weight and all survived until the week 2 after surgery when they were killed. The pyramid bone was removed from eight labyrinthectomized and eight control sites. After 4 weeks of decalcification (Decalcifier solution, Sigma-Aldrich, St Louis, MO, USA, Budapest, Hungary), histological sections were made and studied under the light microscope.

\section{Results}

Animals quickly recovered from anesthesia and were performing the typical neurological symptoms of labyrinthectomy. Skin incisions easily healed; breathing, eating, drinking, and swallowing of the animals were not disturbed from the second day after operation. The weight gain curves of the animals were hardly different from those of their controls. Bilaterally labyrinthectomized rats lost their hearing that could be easily demonstrated (loss of startled movements in response to sudden noise, e.g., clasping the hands). Unilaterally labyrinthectomized animals had decreased the muscle tones at the side of the lesion, with their heads continuously turning toward the lesion. They were moving mostly in unidirectional circuits in their cages, sometimes performing rotational movements around their body axis with directions toward the lesion. When elevated by their tails, the unidirectional rotation was always explicit (Fig. 2d). In a few days, however, they learned to balance using visual and somatic stimuli and led a close to normal life. Nystagmus was subdued during Nembutal anesthesia. After recovery from anesthesia, the classical horizontal nystagmus could be observed with its fast component toward the lesion, but it also diminished as the animals learned to control it relying on visual stimuli. Pathological and histological examinations of decalcified pyramidal bone specimens revealed the empty cochlear cavity with varying 
amount of connective tissue ingrowth. The stria vascularis producing the endolymph has of course been destroyed. The ampullary cristas of the semicircular canals were lowered, they lost their gelatinous cupula coverage and there was a decreased cellularity in the receptor cell layer (Fig. 2b and c).

The technique could be easily taught to persons with some preliminary experience in microscopic surgery. During exploration, special care should be exercised to avoid damage to the retroauricular artery (rostrally), to the external carotid artery branching (below the bulla), and to nerves innervating the laryngeal and neck muscles with incorrectly positioned tissue retractors, while at the same time, ensuring sufficient tissue exploration to avoid damage to muscles while drilling into the bulla. Too extensive clearing of the connective tissue covering the bulla could result unnecessary damage to some facial nerve branches. Exact site, depth, and exerted force of drilling into the cochlear crista should be carefully checked to avoid liquor soaking, basal fracture with subarachnoid bleeding, or damage to the tympanic artery. Adhering to the protocol described above yielded only three animals with unsatisfactory results from a set of 30 .

\section{Discussion}

The applied microsurgical technique ensures a standardized, safe access to the rat cochlea without any damage to important neck, skull, or brain structures. It can be considered as a synthesis of best existing techniques to induce labyrinthectomy in rodents $(1,3,4,7,10,11$, $13,14,18,23-25)$ with a novel standardized microsurgical approach to the mastoid bulla, and to the cochlea. Drilling into the cochlea with subsequent suctioning of the fluid and of cellular contents, as well as the ethanol washes, ensure the destruction of the Corti organ and of the stria vascularis. Loss of the perilymph and endolymph, destruction of the stria vascularis producing the endolymph for the whole labyrinth, and ethanol and arsenite solutions pumped into it immediately and permanently damage the sensitive vestibular function. It needs much less surgical exploration and induces much less damage to surrounding tissues than previous techniques cited above. Loss of cochlear and vestibular functions could be proven by the typical neurological symptoms and also by histology.

\section{Conclusion}

The advantage of the described combined microsurgical-chemical technique to induce labyrinthectomy in rodents is that no additional neural or other damage is made; the animals are able to conduct a fairly normal life. The irreversible vestibular dysfunction will be accompanied with central deafness only. Application of our technique can easily provide the standardized labyrinthectomized rats, popular for neuroplasticity studies; one obstacle of the technique until now being the complicated surgery needed to do the labyrinthectomy. The good shape of the animals makes it possible to do lengthy chronic experiments.

\section{Acknowledgements}

This work was supported by the Hungarian National Grants (OTKA TO 32019 and OTKA TO 42670), the Hungarian Space Agency (MÜI 163), and the Hungarian Kidney Foundation. The authors would like to thank Laszlo Simon, MD, Department of Anatomy, Histology and Embryology, Semmelweis University, Budapest, for his advice. Expert technical assistance is thanked to Ms Oraveczné Ildikó Murányi. 


\section{REFERENCES}

1. Bergquist F, Ruthven A, Ludwig M, Dutia MB: Histaminergic and glycinergic modulation of GABA release in the vestibular nuclei of normal and labyrinthectomised rats. J. Physiol. 577, 857-868 (2006)

2. Cameron SA, Dutia MB: Cellular basis of vestibular compensation: changes in intrinsic excitability of MVN neurons. Neuroreport 8, 2595-2599 (1997)

3. Campos-Torres A, Vidal PP, de Waele C: Evidence for a microglial reaction within the vestibular and cochlear nuclei following inner ear lesion in the rat. Neuroscience 92, 1475-1490 (1999)

4. Cirelly C, Pompeiano M, D'Ascani P, Arrighi P, Pompeiano O: C-fos expression in the rat brain after unilateral labyrinthectomy and its relation to the uncompensated and compensated stages. Neuroscience 70, 515-546 (1996)

5. Curthoys IS: Vestibular compensation and substitution. Curr. Opin. Neurol. 13, 27-30 (2000)

6. Deák A, Bácskai T, Gaál B, Rácz E, Matesz K: Effect of unilateral labyrinthectomy on the molecular composition of perineuronal nets in the lateral vestibular nucleus of the rat. Neurosci. Lett. 513, 1-5 (2012)

7. De Waele C, Abitbol M, Chat M, Menini C, Mallet J, Vidal PP: Distribution of glutamatergic receptors and GAD mRNA-containing neurons in the vestibular nuclei of normal and hemilabyrinthectomized rats. Eur. J. Neurosci. 6, 565-576 (1994)

8. Di Filippo M, Tozzi A, Costa C, Belcastro V, Tantucci M, Picconi B, Calabresi P: Plasticity and repair in the post-ischemic brain. Neuropharmacology 55, 353-362 (2008)

9. Fukushima M, Kitahara T, Takeda N, Saika T, Uno A, Kubo T: Role of cholinergic mossy fibers in medial vestibular and prepositus hypoglossal nuclei in vestibular compensation. Neuroscience 102, 159-166 (2001)

10. Goto MM, Romero GG, Balaban CD: Transient changes in flocculonodular lobe protein kinase C expression during vestibular compensation. J. Neurosci. 17, 4367-4381 (1997)

11. Hitier M, Besnard S, Vignaux G, Denise P, Moreau S: The ventrolateral surgical approach to labyrinthectomy in rats: anatomical description and clinical consequences. Surg. Radiol. Anat. 32, 835-842 (2010)

12. Horii A, Smith PF, Darlington CL: Quantitative changes in gene expression of glutamate receptor subunits/ subtypes in the vestibular nucleus, inferior olive and flocculus before and following unilateral labyrinthectomy in the rat: real-time quantitative PCR method. Exp. Brain Res. 139, 188-200 (2001)

13. Kasri M, Picqet F, Falempin M: Effects of unilateral and bilateral labyrinthectomy on rat postural muscle properties: the soleus. Exp. Neurol. 185, 143-153 (2004)

14. Kim MS, Jin BK, Chun SW, Lee MY, Lee S H, Kim JH, Park BR: Role of vestibulocerebellar N-methylaspartate receptors for behavioral recovery following unilateral labyrinthectomy in rats. Neurosci. Lett. 222, 171-174 (1997)

15. Kitahara T, Takeda N, Kubo T, Kiyama H: An implication of protein phosphatase 2A-beta in the rat flocculus for lesion-induced vestibular plasticity. Acta Otolaryngol. 118, 685-691 (1998)

16. Kitahara T, Takeda N, Saika T, Kubo T, Kiyama H: Effects of MK801 on Fos expression in the rat brainstem after unilateral labyrinthectomy. Brain Res. 700, 182-190 (1995)

17. Lozada AF, Aarnisalo AA, Karlstedt K, Stark H, Panula P: Plasticity of histamine H3 receptor expression and binding in the vestibular nuclei after labyrinthectomy in rat. BMC Neurosci. 5, 32 (2004)

18. Matsuda T, Gotoh TM, Tanaka K, Gao S, Morita H: Vestibulosympathetic reflex mediates the pressor response to hypergravity in conscious rats: contribution of the diencephalon. Brain Res. 102, 140-147 (2004)

19. Monos E, Raffai G, Dörnyei G, Nádasy G, Fehér E: Structural and functional responses of extremity veins to long-term gravitational loading and unloading, lessons from animal systems. Acta Astronaut. 60, 406-414 (2007)

20. Park BR, Choi MA, Hong SM: Temporal changes of calbindin expression in the nodulus following unilateral labyrinthectomy in rats. Neurosci. Lett. 555, 47-50 (2013)

21. Paterson JM, Short D, Flatman PW, Seckl JR, Aitken A, Dutia MB: Changes in protein expression in the rat medial vestibular nuclei during vestibular compensation. J. Physiol. 575, 777-788 (2006)

22. Russell NA, Horii A, Smith PF, Darlington CL, Bilkey DK: Long-term effects of permanent vestibular lesions on hippocampal spatial firing. J. Neurosci. 23, 6490-6498 (2003)

23. Vignaux G, Chabbert C, Gaboyard-Niay S, Travo C, Machado ML, Denise P, Comoz F, Hitier M, Landemore G, Philoxène B, Besnard S: Evaluation of the chemical model of vestibular lesions induced by arsanilate in rats. Toxicol. Appl. Pharmacol. 258, 61-71 (2012)

24. Yamanaka T, Him A, Cameron SA, Dutia MB: Rapid compensatory changes in GABA receptor efficacy in rat vestibular neurones after unilateral labyrinthectomy. J. Physiol. 523, 413-424 (2000)

25. Zheng Y, Horii A, Appleton I, Darlington CL, Smith PF: Damage to the vestibular inner ear causes long-term changes in neuronal nitric oxide synthase expression in the rat hippocampus. Neuroscience 105, 1-5 (2001) 